Pesq. Vet. Bras. 37(9):1015-1024, setembro 2017 DOI: $10.1590 / \mathrm{S} 0100-736 \mathrm{X} 2017000900018$

\title{
Morfologia de órgãos genitais femininos de quelônio semi-aquático Mesoclemmys vanderhaege ${ }^{1}$
}

\author{
Walkiria F. Silva ${ }^{2 *}$, Raissa L. Lima ${ }^{3}$, Juliana N. Pinheiro ${ }^{4}$, Elizângela S. Brito ${ }^{5}$ \\ e Rosa H.S. Ferraz ${ }^{2}$
}

\begin{abstract}
Silva W.F., Lima R.L., Pinheiro J.N., Brito E.S. \& Ferraz R.H.S. 2017. [Morphology of the female genital organs of freshwater turtle Mesoclemmys vanderhaegei.] Morfologia de órgãos genitais femininos de quelônio semi-aquático Mesoclemmys vanderhaegei. Pesquisa Veterinária Brasileira 37(9):1015-1024. Programa de Pós-Graduação em Ciências Veterinárias, Universidade Federal de Mato Grosso, Av. Fernando Corrêa da Costa 2367, Boa Esperança, Cuiabá, MT 78060-900, Brazil. E-mail: walkiria.ferreira@gmail.com

Mesoclemmys vanderhaegei (Testudines: Chelidae) is a freshwater turtle with occurrence in Amazon, Tocantins, Paraguai, Paraná and Uruguai rivers basins. Although according to International Union for Conservation of Nature, it has low risk of extinction, there is an uptade necessity of ecological and biological data. Considering that the management and conservation plans are related to a wide knowledge of reproductive biology, a first macroscopic description about the young and adults females of $M$. vanderhaegei is important. These points were correlated to the specimens size and period of the year. The samples of M. vanderhaegei were collected in Chapada dos Guimarães county, Mato Grosso, Brazil, an area of large natural occurrence of the specimens. Genital organs of seventeen females were fixed in $10 \%$ formalin and then dissected to demonstrate the particularities related to external and internal anatomy. The young and adult $M$. vanderhaegei genitals organs are composed of ovaries and oviducts pairs that dorsolaterally discharge into the cloaca, forming with the ureter, the urogenital papilla. The ovaries are elongated organs with larger cranial and elongated caudal portions. The oviducts, which are in adults very differentiated in its shape and size compared to the young, are long and located laterally to the ovaries. In both age groups, the genital organs are supported by celomatic membrane folds that emerge from the ceiling of the cavity, constituting the mesovary and mesoviduct_In adult females, according to the shape and pattern of the oviduct mucosa, the cranial segment corresponds to the regions of the infundibulum and magnum; the middle segment, the isthmus and the caudal segment identify the uterus and vagina regions. The clitoris is sited on the floor of the urodeum. The carapace linear length and body mass between immature and adult females vary to $134-155.6 \mathrm{~mm}$ e $134.43-365 \mathrm{~g}$ respectively. The main part of young females was captured in rainy period and the adults, with and without eggs, at dry period. The macroscopic characteristics of the M. vanderhaegei genital organs are also observed in others Testudines, with the exception of urogenital papilla and by the clitoris presence.
\end{abstract}

INDEX TERMS: Female genital organs, freshwater turtle, turtle, Mesoclemmys vanderhaegei, anatomy, ovary, oviduct, Testudines.

\footnotetext{
${ }^{1}$ Recebido em 1 de dezembro de 2016.

Aceito para publicação em 1 de março de 2017.

${ }^{2}$ Programa de Pós-Graduação em Ciências Veterinárias (PPGVET), Faculdade de Medicina Veterinária (FAMEV), Universidade Federal de Mato Grosso (UFMT), Av. Fernando Corrêa da Costa 2367, Boa Esperança, Cuiabá, MT 78060-900, Brasil. Pesquisa de doutorado com o apoio Capes. *Autor para correspondência: walkiria.ferreira@gmail.com
}

\footnotetext{
${ }^{3}$ Faculdade de Medicina Veterinária (FAMEV), Universidade Federal de Mato Grosso (UFMT), Av. Fernando Corrêa da Costa 2367, Boa Esperança, Cuiabá, MT 78060-900, Brasil.

${ }^{4}$ Faculdade de Medicina Veterinária, Universidade de Cuiabá (UNIC), Av. Beira-Rio 3100, Jardim Europa, Cuiabá, MT 78000-001, Brasil.

${ }^{5}$ Bolsista Pós-Doutorado CAPES, PPGVET-UFMT, Av. Fernando Corrêa da Costa 2367, Boa Esperança, Cuiabá, MT 78060-900, Brasil.
} 
RESUMO.- O Mesoclemmys vanderhaegei (Testudines: Chelidae) é um quelônio de água doce cuja ocorrência é descrita nas bacias dos rios Amazonas, Tocantins, Paraguai, Paraná e Uruguai. Consta na lista vermelha de espécies ameaçadas da International Union for Conservation of Nature, como espécie de baixo risco, mas que poderá se tornar ameaçada, sendo necessário a atualização de seus dados ecológicos e biológicos. Considerando que planos de manejo e conservação de espécies dependem também de vasto conhecimento sobre a biologia reprodutiva, apresentamos a primeira descrição macroscópica dos órgãos genitais de fêmeas jovens e adultas de $M$. vanderhaegei correlacionando esses achados ao tamanho do espécime e ao período do ano. Exemplares de $M$. vanderhaegei foram coletados no município de Chapada dos Guimarães, área de ampla ocorrência natural da espécie. A descrição dos órgãos genitais foi realizada a partir de 17 fêmeas fixadas em formol a $10 \%$ e dissecadas para evidenciação de particularidades relacionadas à anatomia externa e interna. Os órgãos genitais $M$. vanderhaegei jovens e adultas são constituídos por pares de ovários e ovidutos que desembocam dorsolateralmente na cloaca, formando junto com o ureter a papila urogenital. Os ovários são órgãos alongados, com a extremidade cranial mais larga e caudal afunilada, já os ovidutos são longos e localizados lateralmente aos ovários, sendo em adultas, muito diferenciado na sua forma e tamanho em relação ao das jovens. Em ambas faixas etárias, os órgãos genitais são sustentados por pregas de membrana celomática que emergem do teto da cavidade, constituindo os mesovário e mesoviduto. De acordo com a forma e o padrão de mucosa do oviduto em fêmeas adultas, o segmento cranial corresponde as regiões do infundíbulo e magnum, o segmento médio, ao istmo e no caudal identificam-se as regiões útero e vagina. No assoalho do urodeum aloja-se o clitóris. 0 comprimento retilíneo de carapaça e massa corpórea entre fêmeas imaturas e adultas variam, respectivamente, entre 134-155,6mm e 134,43-365g. A maioria das fêmeas jovens foram capturadas no período chuvoso e as adultas sem e com ovos no período seco. As características macroscópicas dos órgãos genitais observadas em M. vanderhaegei são compartilhadas com outros Testudines, exceto pela papila urogenital e presença do clitóris.

TERMOS DE INDEXAÇ̃̃o: Órgãos genitais femininos, quelônio semi-aquático, Mesoclemmys vanderhaegei, anatomia, ovário, oviduto, Testudines.

\section{INTRODUÇÃO}

Mesoclemmys vanderhaegei é um quelônio de água doce da subordem Pleurodira, família Chelidae (Costa \& Bérnils 2015). Sua ocorrência é descrita nas bacias dos rios Amazonas, Tocantins, Paraguai, Paraná e Uruguai (Marques et al. 2014), abrangendo o norte da Argentina, o Paraguai, o Centro-Oeste e Sudeste do Brasil e Bolívia (Souza 2005, Marques et al. 2014). Em território brasileiro a espécie foi identificada nos estados de Mato Grosso do Sul (Strüssmann et al. 2000), Mato Grosso (Brito et al. 2009b, Brito et al. 2012), Minas gerais (Silveira 2009, Maffei et al. 2016), São Paulo (Souza et al. 2000, Marques et al. 2013, Maffei et al. 2016), Distrito Federal (Brandão et al. 2002), Goiás (Brandão et al. 2002) e Tocantins (Souza et al. 2000, Brandão et al. 2002).
Dados biológicos e ecológicos sobre uma determinada espécie constituem-se no ponto de partida para a determinação do grau de ameaça ao qual ela poderá estar submetida. Segundo Vinke et al. (2013), os participantes do IUCN Red List-ing and Actio Planning Workshop for Chelonians of the Southern Cone" foram unanimes em concordar que somente deveria ser avaliado o status de ameaça das espécies e ser proposto medidas efetivas de conservação, quando mais informações estivessem disponíveis. Especialmente em relação a M. vanderhaegei, Souza (2004) sinaliza que dados relativos a reprodução e alimentação não estão disponíveis, gerando uma lacuna no conhecimento sobre a biologia da espécie, no entanto, alguns estudos relacionados à ecologia e biologia reprodutiva em ambientes naturais (Brito et al. 2009a) e em cativeiro (Corazza \& Molina 2004) foram conduzidos. Atualmente investigações sobre $M$. vanderhaegei são desenvolvidas por dois grupos de pesquisadores, um no estado de São Paulo e outro no Mato Grosso, não obstante, ainda se faz necessária a compreensão de sua biologia no que se refere a distribuição, dieta, reprodução, padrão de atividade e seleção de habitats por essa espécie (Marques et al. 2014).

0 plano de conservação de uma espécie está relacionado a vários parâmetros sendo a biologia reprodutiva de fundamental importância na manutenção das populações naturais. Para M. vanderhaegei, Brito et al. (2009a) descreveram o período de atividade de indivíduos adultos e jovens em ambiente natural, quanto ao comportamento de corte e cópula (dezembro), não sendo observado a presença de ninhos e desova. Em fevereiro de 2015, ovos foram observados no Sudeste do Brasil em indivíduos que desovaram em cativeiro, um dia após a captura em lagos (Maffei et al. 2016). Em animais adultos mantidos em tanques de fibrocimento, Corazza \& Molina (2004) relataram que o acasalamento ocorre sempre dentro d'água, entre os meses de setembro e janeiro, sendo as desovas de janeiro a junho, com pico em abril. Embora se tenha esses parâmetros reprodutivos, informações sobre os locais adequados para nidificação e quais habitats são utilizados pelos jovens (Vinke et al. 2013), assim como as modificações morfofuncionais pelas quais os órgãos genitais são submetidos durante o ciclo reprodutivo, ainda permanecem obscuros. Descrições genéricas para Testudines são encontradas em tratados clássicos (O’Malley 2005, Hildebrand \& Goslow 2006) e também para algumas outras espécies (Kinosternon scorpioides, Podocnemis expansa, Podocnemis unifilis e Phrynops geoffroanus) dessa ordem (Machado Júnior et al. 2006, Cabral et al. 2011, Chaves et al. 2012) sem, no entanto, contrapor esses dados em relação ao período reprodutivo.

Sendo assim, apresentamos a primeira descrição macroscópica dos órgãos genitais de fêmeas jovens e adultas de $M$. vanderhaegei correlacionando esses achados ao tamanho do espécime e ao período reprodutivo, visando estabelecer parâmetros que permitam a compreensão sobre o momento da maturidade sexual na espécie.

\section{MATERIAL E MÉTODOS}

Mesoclemmys vanderhaegei (Bour, 1973) foram capturados e submetidos a eutanásia mediante, respectivamente, licenças emitidas pelo Sistema de Autorização e Informação em Biodiversidade/SISBIO (no 12089-1; no 12089-2; no 25225-2) e aprovação pelo Comité de Éti- 
ca em Pesquisa Animal/UFMT (no 23108026254/08-0). Para tanto, utilizou-se armadilhas tipo covo (funnel trap) e isca de carne bovina colocadas em córregos oligotróficos no município de Chapada dos Guimarães, área de ocorrência natural da espécie, entre os meses de março de 2008 a novembro de 2012 e março e abril de 2016.

Os espécimes capturados foram identificados, mensurados com paquímetro (comprimento retilíneo da carapaça-CRC) e dinamômetro tubular (massa corpórea) e o gênero foi inicialmente estabelecido baseado no comprimento da cauda (Brito et al. 2009b), sendo os espécimes dois, três e quatro identificados como fêmea, somente após a abertura do plastrão por apresentarem CRC inferior a $100 \mathrm{~mm}$, totalizando 17 fêmeas. A sedação foi realizada pela injeção intramuscular de tiletamina associada ao zolazepan na dose de 50mg/kg (Mader 2006) e a eutanásia deu-se pela injeção intracelomática de tiopental sódico a $2 \%(100 \mathrm{mg} / \mathrm{kg})$. Na sequência foi realizada a abertura do plastrão e a fixação dos espécimes por imersão em solução de formol a 10\%. Os órgãos genitais das fêmeas foram dissecados sob lupa circular de luz branca e estereomicroscópio, respectivamente, para evidenciação de suas particularidades da anatomia externa (forma e sintopia dos diferentes segmentos) e interna (padrão de mucosa do oviduto e cloaca). Os espécimes foram agrupados em fêmeas jovens, adultas sem e com ovos, de acordo com as características macroscópicas dos órgãos genitais e informações a cerca dos dados biométricos disponíveis no Quadro 1. A documentação dos achados foi realizada por meio de câmera digital e estereomicroscópio.
Quadro 1. Agrupamento de fêmeas de M. vanderhaegei de acordo com as características de seus órgãos genitais, coletadas em diferentes períodos do ano (PA) e respectivos comprimento retilíneo de carapaça (CRC) e massa corpórea (MC), Chapada dos Guimarães, Mato Grosso, 2016

\begin{tabular}{lcccc}
\hline & Espécimes & CRC $(\mathrm{mm})$ & MC $(\mathrm{g})$ & PA \\
\hline Jovem & 1 & 121,2 & 170,0 & Chuvoso \\
& 2 & 91,5 & 68,0 & Chuvoso \\
& 3 & 90,2 & 67,0 & Chuvoso \\
& 4 & 99,8 & 110,0 & Chuvoso \\
& 5 & 113,3 & 130,0 & Chuvoso \\
& 6 & 134,4 & 220,0 & Chuvoso \\
& 7 & 113,7 & 110,0 & Seco \\
Adulta sem ovos & & $109,2 \pm 16,2$ & $125,0 \pm 55,0$ & \\
& 9 & 182,2 & 440,0 & Seco \\
& 9 & 155,6 & 365,0 & Seco \\
& 10 & 192,5 & 660,0 & Seco \\
Adulta com ovos & 11 & 190,4 & 610,0 & Seco \\
& 12 & 203,0 & 800,0 & Chuvoso \\
& 13 & 179,0 & 490,0 & Seco \\
& 15 & $183,8 \pm 16,2$ & $560,8 \pm 159,6$ & \\
& 16 & 183,0 & 800,0 & Seco \\
& 17 & 193,0 & 1010,0 & Chuvoso \\
& & $196,2 \pm 25,0$ & $695,0 \pm 258,0$ &
\end{tabular}

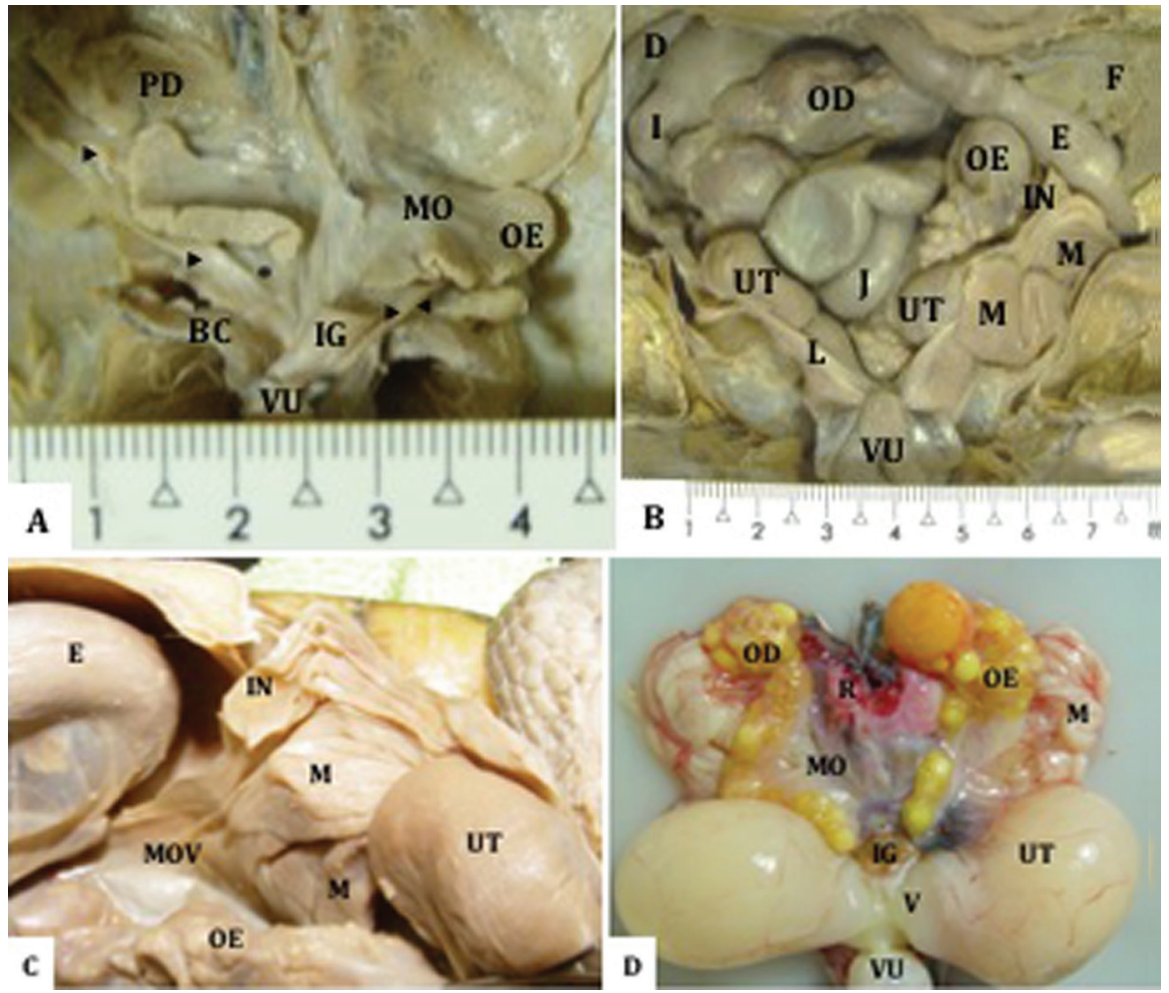

Fig.1. Órgãos genitais femininos de Mesoclemmys vanderhaegei, (A) jovem e (B) adulta sem ovos, fixados in situ. Pulmão direito (PD), mesovário (MO), ovário esquerdo (OE), oviduto ( ), mesoviduto (*), bolsa cloacal (BC), intestino grosso (IG), vesícula urinária (VU). (B) Notar o segmento cranial do oviduto onde, na anatomia interna, há correspondência com as regiões do infundíbulo e magnum, assim como o caudal, está representado pela região denominada de útero. Ovários direito (OD) e esquerdo (OE) com diferentes tamanhos de folículos, duodeno (D), íleo (I), jejuno (J), fígado (F), estômago (E), infundíbulo (IN), magnum (M), útero (UT), ligamento ventral do oviduto (L). A vesícula urinária foi refletida ventralmente, (C) adultas sem e (D) com ovos, respectivamente, peças fixadas in situ e a fresco ex situ. Estômago (E), infundíbulo (IN), magnum (M), mesoviduto (MOV), ovário esquerdo (OE) útero (UT). Observar em (D) os ovários em forma de cacho de uvas com folículos em diferentes estágios de desenvolvimento, a parte cranial e caudal do oviduto, representados respectivamente pelo magnum e útero e vagina. Nota-se o volume correspondente aos ovos no útero. Ovário direito (OD), rim (R), mesovário (MO), intestino grosso (IG), vagina (V) e vesícula urinária (VU) refletida ventralmente. 


\section{RESULTADOS}

Os órgãos genitais de fêmeas dos grupos jovens (Fig.1A), adultas sem (Fig.1B,C) e com ovos (Fig.1D) de Mesoclemmys vanderhaegei são constituídos por pares de ovários e de ovidutos que desembocam, juntamente com os ureteres, na parede dorsolateral da cloaca por meio de evidente projeção na mucosa cloacal denominada de papila urogenital.

Dentre as fêmeas jovens, cinco $(2,3,4,5$ e 7$)$ apresentam órgãos genitais de tamanhos semelhantes e duas outras (1 e 6) maior tamanho, quando observado a proporção que esses órgãos ocupam na cavidade celomática. As demais características são semelhantes entre os sete espécimes.

Os ovários são alongados craniocaudalmente, sendo a extremidade cranial mais larga e a caudal afunilada (Fig.2A) localizada mais próxima à linha mediana, posicionando o maior eixo dos ovários de forma obliqua. Eles ocupam a superfície ventral dos respectivos rins (e seu revestimento celomático), fazendo sintopia aos pulmões (cranialmente), ao oviduto (lateralmente) e ventralmente ao jejuno e cólon descendente, respectivamente, para os ovários direito e esquerdo (Fig.1A). A maior parte da superfície externa desse órgão tem aspecto rugoso devido a presença de folículos ovarianos de dimensões semelhantes, constituídos por conteúdo e invólucro esbranquiçado. A secção longitudinal do ovário, evidencia uma pequena área em sua margem medial, de aspecto esponjoso, a medula ovariana. Nas fêmeas adultas sem ovos, os ovários apresentam dois aspectos: em um espécime (9) a forma é alongada craniocaudalmente, de extremidade cranial mais larga do que a caudal, porém maior que o ovário dos espécimes do grupo anterior (1 e 6) cujos folículos, apesar de pequenos, já mostram diferentes tamanhos entre si (Fig.2B). Nas demais fêmeas desse grupo $(8,10,11,12$ e 13) tanto a forma quanto a superfície ovariana se mostram irregulares, assemelhando-se a um cacho de uva (Fig.1B, D e 2C), com a presença de folículos bem maiores e de diferentes tamanhos que exibem proporcionalmente aumento de vitelo em seu interior (Fig.1D). Os folículos maiores mostram uma faixa esbranquiçada em seu equador denominada de estigma folicular. Nesse grupo os ovários ocupam uma maior área dentro da cavidade celomática. 0 ovário direito relaciona-se ao duodeno descendente e íleo (dorsolateralmente a direita), ao cólon transverso (dorsocranialmente), ao jejuno (ventrocaudalmente) e ao oviduto direito (laterocaudalmente). 0 ovário esquerdo faz sintopia com cólon descendente (dorsalmente), ao estômago (cranialmente) e se interpõem entre as alças jejunais e aos ovidutos direito e esquerdo (Fig.1B). As fêmeas adultas com ovos apresentam também ovários sob dois aspectos: nos espécimes 15 e 17 possuem forma de cacho de uva, cujos folículos ovarianos em diferentes tamanhos estão envolvidos por fina membrana transparente e sustentados por delicada trama de tecido conjuntivo (Fig.1D), já nas fêmeas 14 e 16 as características ovarianas são similares ao espécime oito (adulta sem ovos).

Os ovidutos das fêmeas jovens são estruturas longas e delgadas em forma de estreita fita e parede com espessura homogênea (Fig.1A), direcionando-se dorsoventralmente, margeiam lateralmente os respectivos pulmões e ovários até desembocarem na parede dorsolateral da cloaca. Nas fêmeas adultas sem ovos, o oviduto exibe macroscopicamente três segmentos distintos, o cranial, médio e o caudal (Fig.1B,C). 0 segmento cranial tem forma de fita e é intensamente dobrado sobre si mesmo (Fig.1C,D). A espessura de sua parede aumenta em sentido caudal e varia de delgada e transparente até se tornar mais espessa e opaca, cujo segmento médio (curto e estreito), faz a transição para o caudal. Esse segmento tem forma cilíndrica, de diâmetro uniforme, com escassas dobras, mas com superior espessura de parede, dobrando-se medialmente para transpassar a parede da cloaca, onde desemboca formando a parte genital da papila urogenital, por meio do óstio cloacal. Nas fêmeas adultas com ovos, o oviduto é semelhante, exceto pelo primeiro segmento da região caudal, onde estão localizados os ovos, conferindo a esse local adelgaçamento da parede e diâmetro compatível ao do ovo (Fig.1D). Em cada oviduto observa-se um (espécime 17, Fig.1D), dois (espécimes 14 e 16) e três ovos (espécime 15) totalizando 16 ovos. Os ovos dessa espécie são elipsoides, de casca calcária e branca, no entanto, todos se apresentam em início de formação da casca calcária. Os ovos dos animais 15 e 17 apresentam valores médios para eixo maior e diâmetro de, respectivamente, $3,5 \mathrm{~cm}$ e $2,7 \mathrm{~cm}$ e de $5,3 \mathrm{~cm}$ e $3 \mathrm{~cm}$.

A mucosa do oviduto de fêmeas adultas sem ovos apresenta cinco diferentes regiões. As duas primeiras, infundíbulo e magnum estão localizadas no segmento cranial do oviduto (em forma de fita); a região três, o istmo, corresponde ao segmento médio, enquanto que o útero e a vagina localizam-se no segmento caudal cilíndrico e de parede
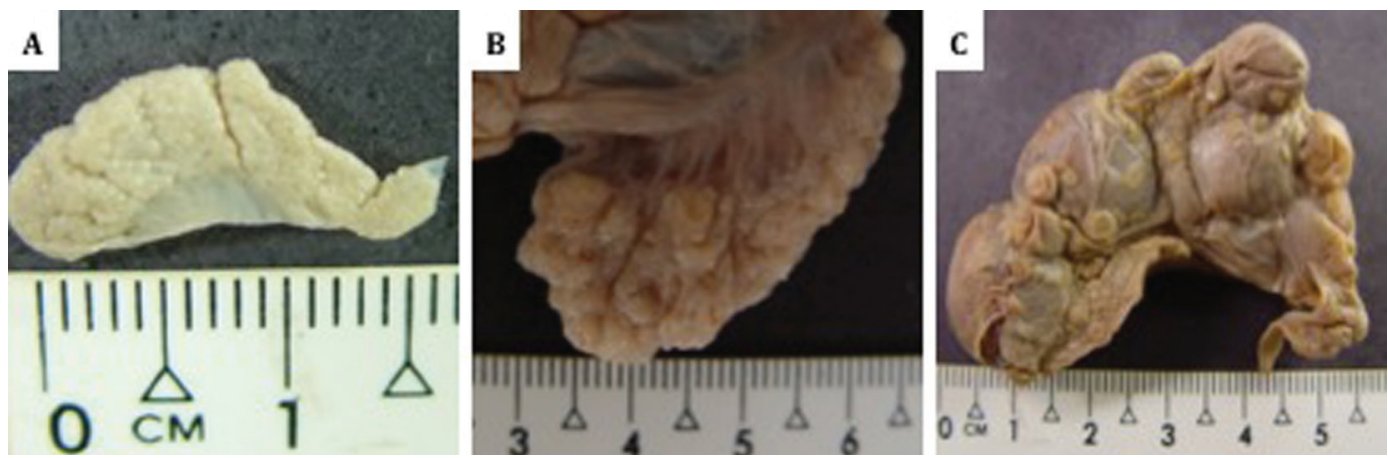

Fig.2. (A) Ovários de Mesoclemmys vanderhaegei fixados in-situ, fêmea jovem e (B) adultas sem e com ovos (B,C). Observar (A) a diferença entre os aspectos homogêneo e (B) heterogêneo dos ovários, assim como, (C) a forma de cacho de uva conferida pelo incremento de vitelo no interior dos folículos a medida que se desenvolvem. 
espessa. 0 infundíbulo possui parede transparente, cuja forma de funil está alojada em sua extremidade cranial e exibe o óstio infundibular em forma de fenda. A mucosa dessa região exibe discreta formação de pregas e o limite entre as regiões do infundíbulo e do magnum não são perceptíveis. No magnum a espessura da parede aumenta deixando de exibir aspecto transparente e a mucosa apresenta pregas longitudinais mais evidentes; na transição entre essas duas regiões (T1), a mucosa possui superfície aveludada (Fig.3A). A mucosa na região do istmo (Fig.3B) exibe intenso pregueamento longitudinal cuja superfície apresenta aspecto variável entre liso a felpudo. 0 útero forma o primeiro e maior segmento da parte caudal do oviduto (Fig.3C). As paredes espessas mostram uma mucosa lisa, brilhante e com pregas dispostas transversalmente na sua porção cranial e longitudinalmente na caudal (Fig.3C). A mucosa da vagina representa a parte genital da papila urogenital, onde o padrão de pregueamento longitudinal se torna mais discreto e em número menor. Nas fêmeas com ovos observam-se características semelhantes, exceto no
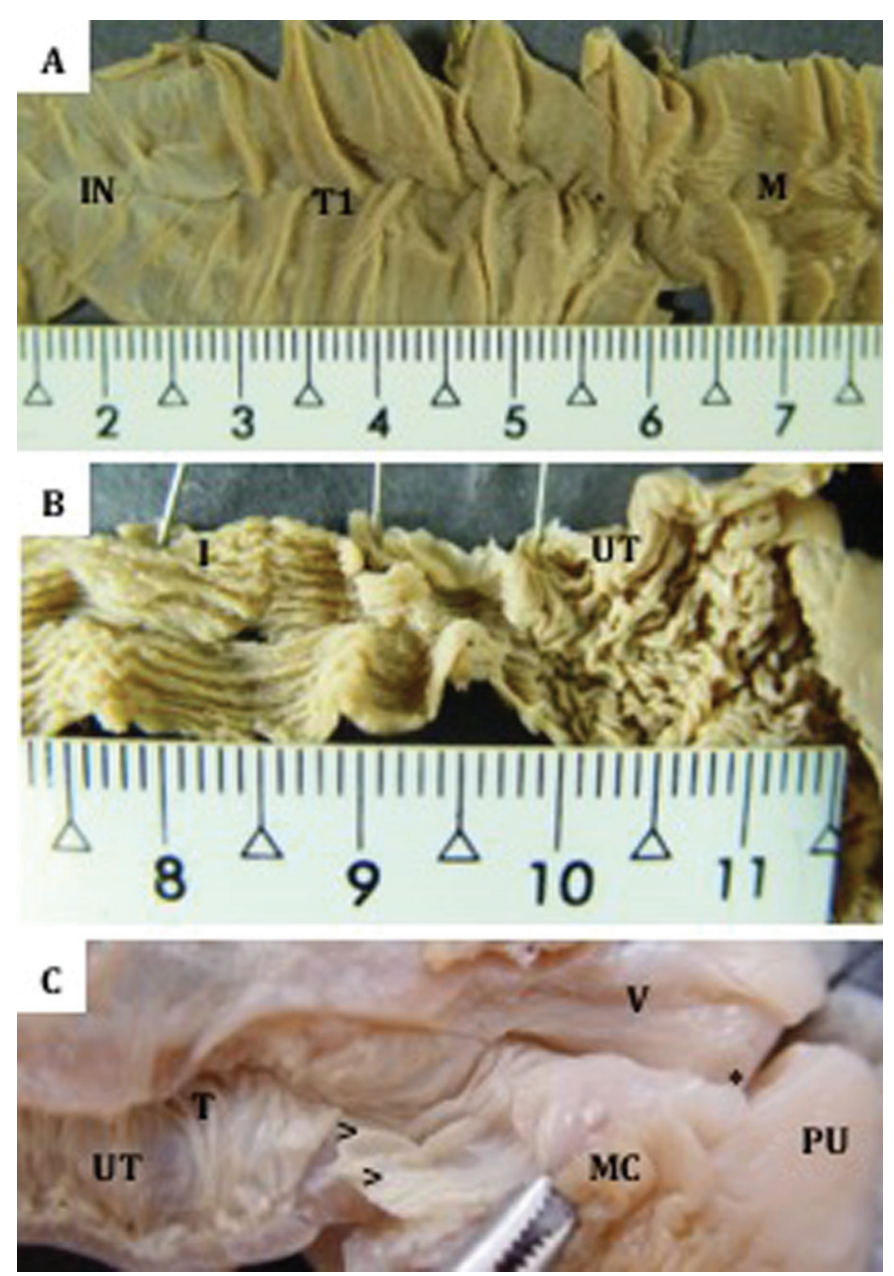

Fig.3 (A) Mucosa do oviduto ex-situ de fêmea adulta sem ovos de Mesoclemmys vanderhaegei, peça fixada, evidencia-se a área de transição (T1) entre as regiões do infundíbulo (IN) e magnum (M). (B) Observar o padrão da mucosa do istmo (I), útero (UT). (C) Nota-se a diferença entre as pregas transversais (T) e longitudinais $(>)$ do útero, vagina $(\mathrm{V})$, mucosa do coprodeum (MC), papila urogenital (PU) e óstio cloacal (*).

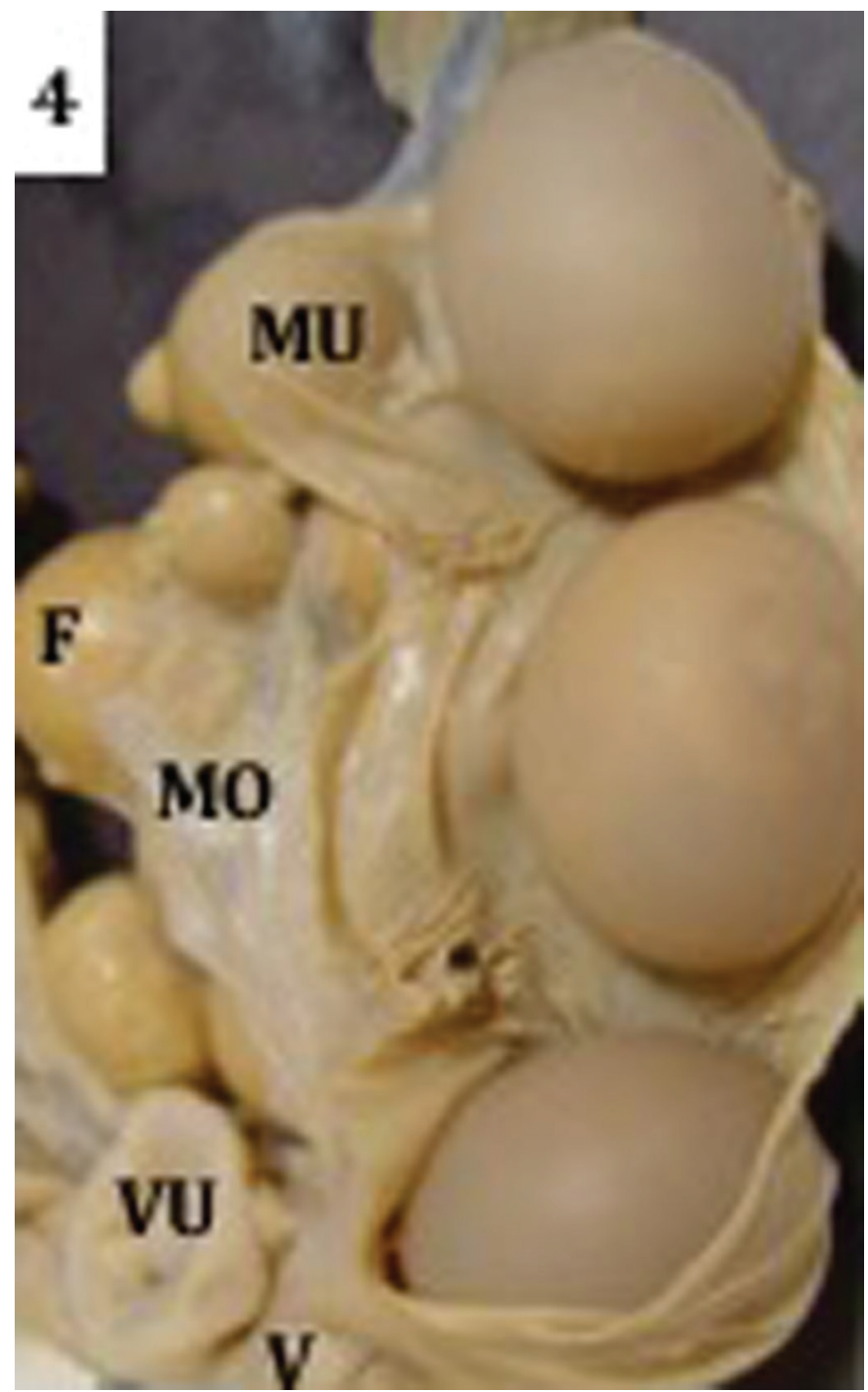

Fig.4. Oviduto esquerdo ex-situ de fêmea com ovos de Mesoclemmys vanderhaegei, peça fixada. Os ovos estão localizados na parte caudal do oviduto (útero), cuja parede se torna adelgaçada e o padrão de pregueamento só é observado na mucosa localizada entre os mesmos. mucosa (MU), mesovário (MO), folículos (F), vesícula urinária (VU), vagina (V).

local ocupado pelos mesmos, onde não se observa pregueamento de mucosa (Fig.4). A luz do oviduto nas fêmeas sem ovos é ampla no infundíbulo, de diâmetro uniforme, porém menor no magnum, estreita no istmo e ampla no útero para se tornar muito reduzida na vagina.

Os elementos de fixação do ovário e oviduto são, respectivamente o mesovário e mesoviduto (Fig.1A-D). 0 mesovário, nos animais jovens, é uma prega de serosa transparente que emerge da superfície ventral do rim, sustentando o ovário pela sua margem medial (Fig.1A), enquanto a margem lateral ovariana é livre. Nas adultas, o mesovário aumenta sua espessura tornando-se esbranquiçado e com a presença de estreitas faixas longitudinais que ocupam toda sua extensão. Em todos os grupos, os ovidutos estão fixados ao teto da cavidade celomática pelo mesoviduto, que varia de fina e transparente a uma espessa membrana, respectivamente, em fêmeas jovens (Fig.1A) e adultas. 


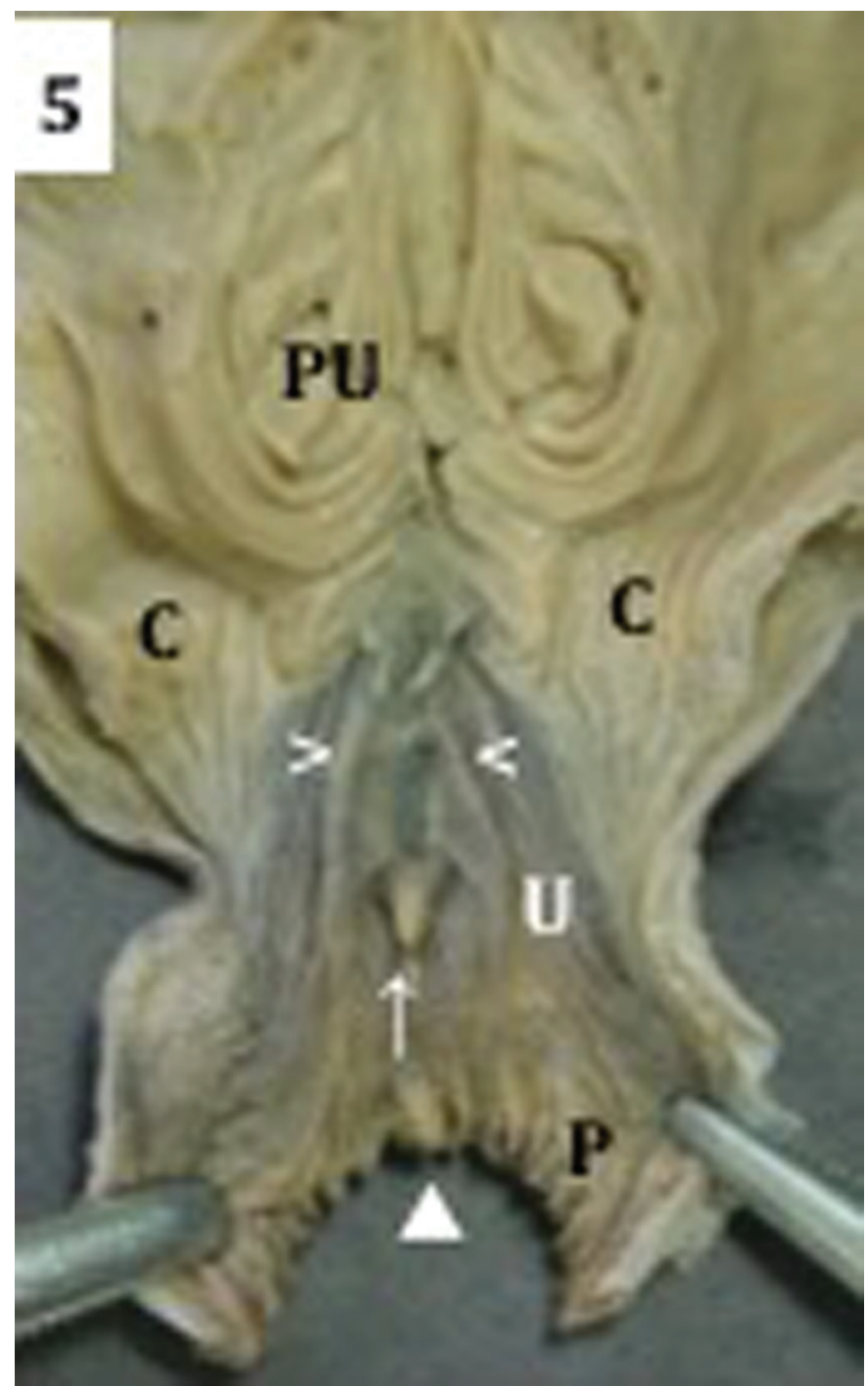

Fig.5. Mucosa do assoalho da cloaca ex situ de Mesoclemmys vanderhaegei, peça fixada. Papilas urogenitais (PU), -coprodeum $(\mathrm{C})$, urodeum $(\mathrm{U})$, clitóris $(\uparrow)$ delimitado pelas pregas laterais $(<)$, proctodeum $(\mathrm{P})$, ventus $(\mathbf{A})$.

A margem dorsal do oviduto é contínua ao mesoviduto e na ventral observa-se um espessamento em forma de cinta, o ligamento ventral do oviduto (Fig.1B), que percorre toda extensão do órgão sustentando sua conformação na fêmea adulta. Essa cinta, na extremidade cranial do oviduto é contínua ao revestimento celomático do teto da cavidade.

Em todas as fêmeas, a cloaca (Fig.5) é um segmento tubular curto constituída por três regiões contiguas que diferem entre si pelo padrão da mucosa e estruturas associadas. A região mais cranial, o coprodeum, constitui-se na porção final do intestino grosso cuja mucosa apresenta pregas longitudinais discretamente sinuosas e que terminam abruptamente na transição ao urodeum. A vesícula urinária abre-se medianamente em posição ventral ao $\mathrm{co}$ prodeum e cranialmente à desembocadura da papila urogenital, marcando o limite cranial do urodeum. A papila urogenital é formada pela projeção do oviduto e ureter ao transpassar as paredes dorsolaterais da cloaca. 0 oviduto abre-se na cloaca por meio do óstio cloacal (Fig.3C) e caudoventralmente a ele, abre-se o óstio do ureter. Ao redor do óstio cloacal, a mucosa apresenta pregas altas que diminuem onde desembocam os ureteres. Nesta região as pregas são lisas, brilhantes e não pigmentadas. A papila urogenital de fêmeas adultas é facilmente observável sendo a das jovens visível somente com auxílio de estereomicroscópio. Caudalmente a papila urogenital, no assoalho do urodeum observam-se duas pregas altas e longas que delimitam uma fossa de onde emerge os pilares (direito e esquerdo) do clitóris (Fig.5). Essas pregas unem-se caudalmente por uma trave transversa de mucosa pigmentada e, são formadas, por colunas de tecido de coloração enegrecida, conforme evidenciado na superfície externa ventral da cloaca. 0 limite entre urodeum e proctodeum não é marcado, e pequenas pregas longitudinais são contínuas entres essas regiões, por outro lado, nota-se uma faixa circular com diminuição da pigmentação diferenciando a mucosa cloacal da epiderme.

\section{DISCUSSÃO}

Diferente das aves (Parizzi et al. 2008, Morais et al. 2012), os quelônios (Machado Júnior et al. 2006, Chaves et al. 2012) apresentam um par de ovários e ovidutos, ficando evidente em fêmeas adultas de $M$. vanderhaegei que a atividade ovariana é bilateral, devido a presença de pares de ovos nos ovidutos (útero) que correspondem a atividade dos ovários direto e esquerdo.

As fêmeas jovens de Mesoclemmys vanderhaegei apresentam ovários e ovidutos de pequenas proporções e aspecto homogêneo, sugerindo ausência de atividade ovariana (ausência de foliculogênese), mesmo para os espécimes que apresentam ovários e ovidutos maiores $(1 ; 6)$, há manutenção do aspecto homogêneo relacionados ao tamanho dos folículos e ao oviduto. As características ovarianas relacionadas aos seus limites (cranial e caudal, medial e lateral) indicam que os mesmos são imaturos e semelhantes às características apresentadas nas descrições de ovários de Podocnemis expansa e Podocnemis unifilis (Malvásio et al. 2012) e em aves (Komárek \& Procházková 1970), ambos em animais recém-eclodidos. Uma fêmea adulta sem ovos (2), embora assim considerada por ter um oviduto mais desenvolvido, apresenta sintopia semelhante à dos ovários das fêmeas jovens.

Durante o ciclo ovariano, os ovários têm sua sintopia modificada devido ao recrutamento folicular, que aumenta tanto o número quanto o tamanho dos folículos (foliculogênese), se interpondo entre outros órgãos que até então não estabeleciam relação de vizinhança, condição também citada em K. scorpioides (Machado Júnior et al. 2006, Chaves et al. 2012) e em aves (Morais et al. 2012). Apesar dos ovários das fêmeas adultas com ovos (14 e 16) apresentarem estrutura e sintopia semelhantes ao espécime 9 (adulta sem ovos), a presença de ovos em processo de formação de casca calcária, tanto pode sugerir o término de um ciclo quanto o início de outro, visto que folículos em diferentes tamanhos sugerem foliculogênese, mesmo que ainda não tenham modificado a forma inicial do ovário. Já nas fêmeas 15 e 17 é possível inferir que haverá um novo ciclo ovariano devido ao aspecto de cacho de uva dos ovários, 
exibindo, portanto, diferentes graus de armazenamento de vitelo (vitelogênese) e, embora seja necessário se realizar coleta de dados de modo contínuo, esta é uma evidência de que poderá haver mais de uma oviposição por período reprodutivo em ambiente natural, apesar da variável disponibilidade de alimentos em animais de vida livre. Essa característica também havia sido relatada para as espécies M. vanderhaegei (Corazza \& Molina 2004) e Hydromedusa tectifera (Chinen et al. 2004), cujas condições de cativeiro propiciam relativa constância alimentar.

Em ambiente natural, a definição do gênero ocorre pela observação dos caracteres sexuais secundários como comprimento e espessura da cauda, sendo mais curta e delgada nas fêmeas em relação aos machos (Brito et al. 2009b). Quando os animais apresentam CRC inferior a $100 \mathrm{~mm}$, eles são caracterizados como gênero indeterminado (Brito et al. 2009 b), como ocorre com alguns espécimes jovens (2, 3, 4) de $M$. vanderhaegei. Nas demais fêmeas jovens, o gênero foi identificado no momento da coleta por apresentarem CRC acima de $100 \mathrm{~mm}$ permitindo a diferenciação das características relativas a cauda. Apesar disso, os resultados macroscópicos sinalizam que, se há atividade ovariana (foliculogênese) nos animais desse grupo, ela ainda não seria suficiente para modificar o aspecto macroscópico do ovário e oviduto, mesmo nos exemplares maiores (1 e 6) onde o que diferiu nos mesmos foi apenas o tamanho dos órgãos sem qualquer outra modificação. É provável que essa diferenciação de gênero esteja associada ao treinamento do observador, pois além de não haver características macroscópicas que possam evidenciar a atividade ovariana, não foi possível utilizar métodos de maior acurácia a fim de se inferir se essa percepção está ou não associada a caracteres sexuais secundários oriundos de estímulo hormonal. Por outro lado, a menor fêmea adulta ( $9=155,6 \mathrm{~mm}$ e $365 \mathrm{~g})$ mostra diferenciação de tamanho de folículos (foliculogênese), mas ovários de tamanho semelhante ao das fêmeas jovens, no entanto, o oviduto desse espécime, apresenta caraterísticas que sugerem ter havido atividade reprodutiva prévia, não havendo neste grupo, dificuldade de identificação do gênero, seja pelo CRC do animal ou pela observação de caracteres sexuais secundários. Considerando o maior espécime de fêmeas jovens ( $6=134 \mathrm{~mm}$ e $220 \mathrm{~g}$ ) e a menor fêmea adulta sem ovos ( $2=155,6 \mathrm{~mm}$ e $365 \mathrm{~g})$ pode-se inferir que a maturidade sexual ocorra em animais que apresentem CRC entre 134 a 155,6mm, o que é corroborado pelos dados obtidos em $M$. vanderhaegei cativos, onde a menor fêmea apresenta $152 \mathrm{~mm}$ de comprimento de carapaça, apesar das fêmeas capturadas imediatamente após a desova possuírem 148mm (Corazza \& Molina 2004). Considerando o intervalo de CRC (134 a $155,6 \mathrm{~mm})$ proposto para o início da maturidade sexual nessa espécie, os dados apresentados sugerem que ela esteja mais próxima do limite superior, mas é provável que não seja relacionado ao CRC, mas sim a massa corpórea, portanto, a maturidade sexual ocorreria em fêmeas entre 220 a 365g. Neste estudo, fêmeas sem ovos, que apresentaram características macroscópicas de atividade reprodutiva tiveram massa corpórea mínima de $365 \mathrm{~g}$, sendo mais precoces do que aquelas em cativeiro cujos valores mínimo e máximo apresentados por Corazza \& Molina (2004) foram, respectivamente, de 507 e $1610 \mathrm{~g}$.

Os ovidutos das fêmeas jovens não apresentam diferenciação. Esse aspecto homogêneo sugere ausência de atividade hormonal sobre esse órgão alvo, portanto, não é possível inferir diferentes regiões a um órgão imaturo, de modo semelhante ao observado em fêmeas recém-eclodidas de $P$. expansa e $P$. unifilis (Malvásio et al. 2012). As fêmeas adultas, apresentam forma e tamanho diferenciados, assim como modificação na espessura da parede, que se mostra progressiva em sentido do fluxo de migração cloacal, condição sugestiva de atividade reprodutiva, permitindo a identificação de diferentes regiões, cuja relações filogenéticas entre répteis e aves possibilitam a comparação.

King (1993a-b) padroniza os termos referente as diferentes regiões do oviduto como infundíbulo, magnum, istmo, útero e vagina, no entanto, em répteis Palmer \& Guillette (1988) e Girling (2002) adotam o termo "uterine tube", mas Girling cita sinonímias ("tuba, glandular region, albumen-secreting portion") para se referir a região do magnum nas aves. Apesar dessas cinco regiões serem classicamente reconhecidas em $K$. scorpioides (Machado Júnior et al. 2006) e em aves (King 1993a), em M. vanderhaegei, não há correspondência direta entre elas e os segmentos macroscópicos cranial e caudal do oviduto nas fêmeas adultas sem ovos, exceto para o segmento médio que corresponde a região do istmo.

Em análise ao aspecto externo do oviduto de $M$. vanderhaegei, o segmento cranial tem parede fina, em forma de fita intensamente dobrada sobre si mesmo, conferindo um tamanho superior em relação a área que ocupa na cavidade celomática, graças ao recurso de dobramento. Abriga dois padrões distintos de mucosa, definidos como regiões do infundíbulo e magnum (King 1993a, Machado Júnior et al. 2006), portanto, esse segmento somente foi definido após a observação da mucosa. A forma de funil é apropriada para a captação de oócitos, cuja abertura em forma de fenda parece ser uma característica de $M$. vanderhaegei diferindo de Gopherus polyphemus (Palmer \& Guillette, 1988) e K. scorpioides (Malvásio et al. 2012). 0 maior comprimento da região magnum, permite que o albúmen seja acrescido ao futuro ovo, condição encontrada em Testudines e Crocodylia, mas não em Squamata (Girling 2002). Entre essas regiões, a transição é marcada por três modificações, a perda da transparência da parede do oviduto, o incremento das pregas longitudinais e a mudança no aspecto da mucosa.

O segmento médio do oviduto denominado de istmo, além de mais estreito, possui padrão variável de mucosa, podendo assemelhar-se as regiões contíguas a ele. Essa região também foi observada em $K$. scorpioides (Machado Júnior et al. 2006), exceto a transição, pois nessa espécie a mucosa não foi caracterizada.

O segmento caudal, corresponde as regiões do útero e vagina, apesar de não haver nas fêmeas adultas sem ovos, variação de diâmetro entre elas. A parede da região do útero é mais espessa em relação as regiões que a antecede, característica compartilhada por K. scorpioides (Malvásio et al. 2012) e Gopherus polyphemus (Palmer \& Guillette, 1988) sendo, nessa última espécie, de diâmetro maior devi- 
do também ao aumento da luz. Apesar de ter sido observado em M. vanderhaegei o aumento de diâmetro nas fêmeas que apresentavam ovos nessa região, a parede se apresentava adelgaçada. 0 padrão de mucosa do útero exibe pregas transversais e longitudinais e remete não só ao aumento de superfície de contato, como também ao controle do fluxo de migração cloacal. Enquanto em todas as outras regiões, as pregas longitudinais favorecem o fluxo no sentido cloacal, a primeira região do útero, com suas pregas transversais, possivelmente retarda esse fluxo (King 1993a), já que o útero tem comprimento bem inferior ao o magnum, apesar de em ambos, aos autores os caracterizarem como segmentos secretores (Palmer \& Guillette 1988, Girling 2002, Firmiano et al. 2012). A última região do oviduto, a vagina, corresponde a parte genital da papila urogenital em M. vanderhaegei, que semelhante ao citado por Sánchez-Martínez et al. (2007) em Mabuya sp., desemboca na parede dorsolateral da cloaca. É uma região curta e de espessura superior a do útero, que juntamente com o ureter projeta-se na luz do urodeum formando o que designamos de papila urogenital. Apesar de não haver referência a desembocadura do oviduto e ureter em uma única estrutura (papila urogenital) em K. scorpioides (Machado Júnior et al. 2006), assim como, Girling 2002, esses autores afirmam a condição de esfíncter da vagina, corroborando a observação de sua consistência firme em M. vanderhaegei. Sánchez-Martínez et al. (2007) também se referem a essa condição de esfíncter, mas ao representar o complexo vagina-cloaca de Mabuya sp. fazem menção a intrusão da vagina na parede da cloaca, designado essa estrutura como tubérculo genital. Já Gabe \& Saint-Girons (1965) denominaram-na de papila genital. Esses termos nos levam a inferir que a abertura dos ureteres na cloaca, não ocorre juntamente ao oviduto, a semelhança do que ocorre em $M$ vanderhaegei que também foi descrito em várias espécies de Pleurodira por Rosa et al. (2011).

Tanto em fêmeas jovens como em adultas os ovários e ovidutos são sustentados, respectivamente, pelo mesovário e mesoviduto como descrito para as espécies $P$. expansa, $P$. unifilis (Malvásio et al. 2012) e K. scorpioides (Machado Júnior et al. 2006, Chaves et al. 2012). No entanto, esses autores não fazem menção as modificações dessa estrutura, que variam de acordo com a condição funcional da fêmea, atingindo sua maior espessura nas fêmeas com ovos, sugerindo que esses elementos de sustentação, assim como os ovidutos, estão sujeitos a ação hormonal, muito perceptível no incremento que sofre o ligamento ventral do oviduto, em forma de cinta nas fêmeas adultas.

A cloaca de M. vanderhaegei apresenta os compartimentos coprodeum, urodeum e proctodeum, sendo esse arranjo um consenso na literatura em répteis (Sánchez-Martínez et al. 2007, Rosa et al. 2011, Gredler et al. 2014) e aves (King 1993b). Apesar de estudos anteriores definirem a cloaca como um compartimento comum aos sistemas digestório e urogenital (Machado Júnior et al. 2006, Pinheiro et al. 2010), pouca atenção foi dada ao urodeum, em cujo assoalho repousa o clitóris, não mencionado em $K$. scorpioides (Machado Júnior et al. 2006) e tampouco em P. expansa, $P$. unifilis, Chelus fimbriatus e Phrynops geoffroanus (Rosa et al. 2011).
Exceto o espécime sete que foi capturado durante o período seco, as demais fêmeas jovens de M. vanderhaegei foram capturadas no período chuvoso. Segundo Souza (2004) parece haver um sincronismo entre as fases de nidificação e nascimento de filhotes com as condições climáticas locais. É fato que essas espécies têm em comum o período chuvoso, parecendo ser essa época, o momento mais adequado para o nascimento dos filhotes. Isso também é observado em P. sextuberculata (Haller e Rodrigues, 2006), Hydromedusa maximiliani (Famelli et al. 2014) e P. expanda e P. unifilis (Malvásio et al. 2012) de vida livre e em Hydromedusa tectifera (Chinen et al. 2004) em cativeiro.

0 único relato de período de incubação em $M$. vanderhaegei foi em cativeiro e descreve a eclosão de um único filhote após 300 dias de incubação, acasalamento entre setembro e janeiro e desovas de janeiro a junho (Corazza \& Molina 2004) e, em ambiente natural, a cópula foi observada em dezembro (Brito et al. 2009a). De acordo com esses parâmetros podemos verificar que entre o acasalamento e a desova houve um intervalo de quatro a cinco meses, levando-nos a supor que o período de incubação ocorra no final do período chuvoso e início do seco. É possível que isso explique a maioria das fêmeas com ovos terem sido capturadas durante a estação seca, assim como, as fêmeas sem ovos apresentarem nessa mesma época, ovários em fase foliculogênica e vitelogênica, sugerindo que o período reprodutivo da espécie possa ocorrer na estação seca.

\section{CONCLUSÕES}

Os órgãos genitais de fêmeas de Mesoclemmys vanderhaegei jovens e adultas são constituídos por pares de ovários e ovidutos sustentados, respectivamente, pelo mesovário e mesoviduto que apresentam diferenças entre jovens, adultas sem e com ovos.

Ovários de fêmeas jovens apresentam aspecto homogêneo e os das adultas apresentam variáveis estágios de foliculogênese e vitelogênese. Nas fêmeas jovens, eles apresentam extremidade cranial larga e caudal afunilada, em adultas os ovários possuem formato de cacho de uva.

Os ovidutos são retilíneos em animais jovens e em adultos diferenciam-se em três segmentos macroscópicos: um cranial, em forma de fita com um funil em sua extremidade cranial, um médio curto e estreito e outro caudal, cilíndrico e de parede espessa (exceto nas fêmeas com ovos) que desemboca na parede dorsolateral do urodeum formando a papila urogenital.

O segmento cranial do oviduto dobra-se sobre si mesmo cuja espessura aumenta em sentido cloacal, sendo transparente em sua extremidade cranial. Sua forma e o padrão da mucosa correspondem as regiões do infundíbulo e magnum. 0 segmento médio corresponde ao istmo e no segmento caudal identificam-se as regiões útero e vagina.

A cloaca é constituída pelas regiões coprodeum, urodeum e proctodeum e do assoalho do urodeum emerge o clitóris.

A maioria das fêmeas jovens foram capturadas no período chuvoso e as adultas sem e com ovos no período seco.

De acordo com a biometria da maior fêmea jovem e a menor fêmea adulta, sugere-se que a maturidade sexual em 
fêmeas de $M$. vanderhaegei, ocorra em animais com CRC de 134 a 155,6mm e massa corpórea de 220 a 365g.

\section{REFERÊNCIAS}

Bour R. 1973. Contribution in Conna issance of Phrynops nasutus (Schweigger, 1812) and Phrynops tuberculatus (Luederwaldt, 1926). Description a New subspecies Originarie of the Paraguay, Phrynops tuberculatus vanderhaegei. (Testudinata-Pleurodira-Chelidae). Newsl. Zool. Soc. Fr., Paris, 98(1):175-190.

Brandão R.A., Zerbini G.J., Sebben A. \& Molina F.B. 2002. Notes on distribution and habitats of Acanthochelys spixii and Phrynops vanderhaegei (Testudines, Chelidae) in central Brazil. Bolm Asoc. Herpetol. Esp., Madri. 3(1/2):11-15.

Brito E.S., Strüssmann C., Kawashita-Ribeiro R.A., Morais D.H., Ávila R.W. \& Campos V.A. 2012. New records and distribution extensions of three species of Mesoclemmys Gray, 1863 (Testudines: Chelidae) in Mato Grosso state, Brazil, with observations on terrestrial movements. Check List 8(2):294-297.

Brito E.S., Strüssmann C. \& Baicere-Silva C.M. 2009a. Courtship behavior of Mesoclemmys vanderhaegei (Bour, 1973) (Testudines: Chelidae) under natural conditions in the Brazilian Cerrado. Herpetol. Notes 2:67-72.

Brito E.S., Strüssmann C. \& Penha J.M.F. 2009b. Population Structure of Mesoclemmys vanderhaegei (Bour, 1973) (Testudines: Chelidae) in the Cerrado of Chapada dos Guimarães, Mato Grosso, Brazil. Biota Neotrop. 9(4):245-248

Cabral S.R.P., Santos L.R.S., Franco-Belussi L., Zieri R., Zago C.E.S. \& Oliveira C. 2011. Anatomy of the male reproductive system of Phrynops geoffroanus (Testudines, Chelidae). Acta Scient., Biol. Sci. 33(4):487-492.

Chaves E.P., Oliveira S.C.R., Araújo L.P.F., Oliveira A.S., Miglino M.A., Abreu-Silva A.L., Melo F.A. \& Sousa A.L. 2012. Morphological aspects of the ovaries of turtle Kinosternon scorpioides raised in captivity. Pesq. Vet. Bras. 32(7):667-671.

Chinen S., Lisboa C.S. \& Molina F.B. 2004. Biologia reprodutiva de Hydromedusa tectifera em cativeiro (Testudines, Chelidae). Arqs Inst. Biológico, São Paulo, 71(Supl.):1-749.

Corazza S.S. \& Molina F.B. 2004. Biologia reprodutiva e conservação ex-situ de Bufocephala vanderhaegei (Testudines, Chelidae). Arqs Inst. Biológico, São Paulo, 71:210-409.

Costa H.C. \& Bérnils R.S. 2015. Répteis brasileiros: Lista de espécies 2015. Herpetologia Brasileira 4(3). Disponível em <http://www.sbherpetologia.org.br/images/LISTAS/2015-03-Repteis.pdf> Acesso em 20 out. 2016.

Famelli S., Adriano L.R., Pinheiro S.C.P., Souza F.L. \& Bertoluci J. 2014. Reproductive Biology of the Freshwater Turtle Hydromedusa maximiliani (Chelidae) from Southeastern Brazil. Chelonian Conserv. Biol. 13(1):81-88.

Firmiano E.M.S., Cardoso N.N., Santos M.A.J., Sousa B.M., Nascimento A.A. \& Pinheiro N.L. 2012. Histology and Histochemistry of the Oviduct of the Neotropical Tortoise Phrynops geoffroanus (Schweigger, 1812). J. Cytol. Histol. 3(7). Disponível em <http://dx.doi.org/10.4172/21577099.1000164> Acesso em 22 nov. 2016.

Gabe M. \& Saint-Girons H. 1965. Contribution a la morphologie comparée du cloaque et des glandes épidermoïdes da la région cloacale chez les lépidosauriens. Mémoires du Muséum National D’Histoire Naturalle, Série A, Zoologie XXXIII(4):151-292.

Girling J.E. 2002. The reptilian oviduct: a review of structure and function and directions for future research. J. Exp. Zool. 293:141-170.

Gredler M.L., Larkins C.E., Leal F., Lewis A.K., Herrera A.M., Perriton C.L., Sanger T.J. \& Cohn M.J. 2014. Evolution of external genitalia: insights from reptilian development. Sex. Dev. 8:311-326.

Haller E.C.P. \& Rodrigues M.T. 2006. Reproductive biology of the six-tuberculed Amazon river turtle Podocnemis sextuberculata (Testudines: Podocnemididae), in the Biological Reserve of Rio Trombetas, Pará, Brazil. Chelonian Conserv. Biol. 5(2):280-284.

Hildebrand M. \& Goslow G. 2006. Órgãos genitais e Ductos Urogenitais, p.284-293. In: Ibid. (Eds), Análise da Estrutura dos Vertebrados. $2^{2}$ ed. Atheneu, São Paulo.

King A.S. 1993a. Organa genitalia feminina, p.370-389. In: Baumel J.J., King A.S., Breazile J.E., Evans H.E. \& Berge C.V. (Eds), Handbook of Avian Anatomy: Nomina Anatomica Avium. 2nd ed. Nuttall Ornithological Club, Cambridge, Massachusetts.

King A.S. 1993b. Cloaca, p.390-397. In: Baumel J.J., King A.S., Breazile J.E., Evans H.E. \& Berge C.V. (Eds), Handbook of Avian Anatomy: Nomina Anatomica Avium. 2nd ed. Nuttall Ornithological Club, Cambridge, Massachusetts.

Komárek V. \& Procházková E. 1970. Growth and diferenciation of the ovarian folicules in the pós-natal development of the chicken. Acta Vet. Brno 39:11-16.

Machado Júnior A.A.N., Sousa A.L., Santos F.C.F. \& Pereira J.G. 2006. Morfologia dos órgãos genitais femininos do muçuã (Kinosternon scorpioides). Arch. Vet. Sci. 11(2):25-29.

Mader D.R. 2006. Euthanasia, cap. 5(33), p.564-548. In: Ibid. (Ed.), Reptile Medicine and Surgery. 2nd ed. Elsevier Saunders, Missouri.

Maffei F., Nascimento B.T.M., Moya G.M. \& Donatelli R.J. 2016. New distribution records of Mesoclemmys vanderhaegei (Testudines: Chelidae) from southeastern Brazil, including observations on reproduction. J. Threatened Taxa 8:9322-9326.

Malvásio A., Nascimento-Rocha J.M., Santos H.D., Ataídes A.G. \& Portelinha T.C.G. 2012. Morfometria e histologia das gônadas de machos e fêmeas recém- eclodidos de Podocnemis expansa e Podocnemis unifilis (Testudines, Podocnemididae). Acta Scient., Biol. Sci. 34(1):105-112.

Marques T.S., Lara N.R.F., Bassetti L.A.B., Ferronato B.O., Malvásio A. \& Verdade L.M. 2013. Population structure of Mesoclemmys vanderhaegei (Testudines, Chelidae) in a silvicultural system in southeastern Brazil. Herpetol. Notes 6:179-182.

Marques T.S., Böhm S., Brito E.S., Cabrera M.R. \& Verdade L.M. 2014. Mesoclemmys vanderhaegei (Bour, 1973) - Vanderhaege's Toad-headed Turtle, Karumbé-hy. In: Rhodin A.G.J., Pritchard P.C.H., Van Dijk P.P., Saumure R.A., Buhlmann K.A., Iverson J.B. \& Mittermeier R.A. (Eds), Conservation Biology of Freshwater Turtles and Tortoises: a compilation project of the IUCN/SSC Tortoise and Freshwater Turtle Specialist Group. Chelonian Research Monographs 5(7):083.1-8. doi:10.3854/crm.5.083.vanderhaegei. v1.2014, <http://www.iucn-tftsg.org/cbftt/>

Morais M.R.P.T., Velho A.L.M.C.S., Dantas S.E.S. \& Fontenele-Neto J.D. 2012. Morfofisiologia da reprodução das aves: desenvolvimento embrionário, anatomia e histologia do sistema reprodutor. Acta Vet. Bras. 6(3):165176.

O’Malley B. 2005. Tortoises and turtles, p.40-56. In: Ibid. (Ed.), Clinical Anatomy and Physiology of Exotic Species. Elsevier Saunders, Philadelphia.

Palmer B.D. \& Guillette L.J. 1988. Histology and functional morphology of the female reproductive tract of the tortoise Gopherus polyphemus. Am. J. Anat. 183:200-221.

Parizzi R.C., Santos J.M., Oliveira M.F., Maia M.O., Sousa J.A., Miglino M.A. \& Santos T.C. 2008. Macroscopic and Microscopic anatomy of the oviduct in the sexually mature rhea (Rhea americana). Anat. Histol. Embryol. 37:169-176.

Pinheiro J.N., Godoy I., Brito E.S., Strüssmann C. \& Ferraz R.H.S. 2010. Macroscopia do aparelho digestório do cágado sul-americano Mesoclemmys vanderhaegei (Bour, 1973). Braz. J. Vet. Res. An. Sci. 47(6):429-438.

Rosa M.A., Santos A.J.Q., Menezes L.T., Kaminishi A.P.S., Leonardo T.G. \& D’Aparecida N.S. 2011. Anatomia da cloaca de algumas espécies de Pleurodira (Reptilia: Testudines). PUBVET, Londrina, 5(11), ed. 158, art. 1070. Disponível em <http://www.cabi.org/cabdirect/FullTextPDF/2011/20113177274.pdf> Acesso em 26 jun. 2012.

Sánchez-Martínez P.M., Ramírez-Pinilla M.P. \& Miranda-Esquivel D.R. 2007. Comparative histology of the vaginal- cloacal region in Squamata and its phylogenetic implications. Acta Zool., Stockholm, 88:289-307.

Silveira A.L. 2009. Mesoclemmys vanderhaegei. Minas Gerais, Brasil. Herpetol. Review 40(2):235-236.

Souza F.L. 2004. Uma revisão sobre padrões de atividade, reprodução e ali- 
mentação de cágados brasileiros (Testudines, Chelidae). Phyllomedusa 3(1):15-27.

Souza F.L., Martins M. \& Sawaya R.J. 2000. A new record and observations of vanderhaege's toad-headed turtle, Phrynops vanderhaegei (Testudines, Chelidae) in Brazil. Boln Asoc. Herpetol. Esp. 11(12):85-88.

Souza F.L. 2005. Geographical distribution patterns of South America sidenecked turtles (Chelidae), with emphasis on Brazilian species. Revya Esp. Herpetol. 19:33-46.

Strüssmann C., Prado C.P.A. \& Uetanabaro M. 2000. Amphibians and rep- tiles of selected localities in the Southern Pantanal floodplains and surrounding cerrado, Mato Grosso do Sul, Brasil, p.98-102. In: Willink P.W., Chernoff B., Alonso L.E., Montambault J.R. \& Lourival R. (Eds), A Biological Assessment of the Aquatic Ecosystems of the Pantanal, Mato Grosso do Sul, Brasil. RAP Bulletin of Biological Assessment 18m, Conservation International, Washington, DC.

Vinke T., Vinke S. \& Köhler G. 2013. What is known about Mesoclemmys vanderhaegei (Bour, 1973): a systematic review of the available literature. Paraquaria Nat. 1(2):21-31. 